\title{
Calculating Operational Patterns for Electric Vehicle Charging on a Real Distribution Network Based on Renewables' Production
}

\author{
Stavros Lazarou ${ }^{0}$, Vasiliki Vita, Christos Christodoulou and Lambros Ekonomou* \\ Department of Electrical and Electronic Engineering Educators, School of Pedagogical \& Technological \\ Education (ASPETE), Heraklion Attikis, 14121 Athens, Greece; slazarou@ta.aspete.gr (S.L.); \\ vasvita@aspete.gr (V.V.); christ_fth@uth.gr (C.C.) \\ * Correspondence: leekonom@gmail.com; Tel.: +30-210-2896-927
}

Received: 30 July 2018; Accepted: 7 September 2018; Published: 11 September 2018

\begin{abstract}
The connection of electric vehicles to distribution networks has been an emerging issue of paramount importance for power systems. On one hand, it provides new opportunities for climate change mitigation, if electric energy used for charging is produced from zero emission sources. On the other hand, it stresses networks that are now required to accommodate, in addition to the loads and production from distributed generation they are initially designed for, loads from electric vehicles charging. In order to achieve maximum use of the grid without substantially affecting its performance, these issues have to be addressed in a coordinated manner, which requires adequate knowledge of the system under consideration. It is advantageous that electric vehicle charging can be controlled to a certain degree. This research provides better understanding of real distribution networks' operation, proposing specific operational points through minimizing electric vehicle charging effects. The probabilistic Monte Carlo method on high performance computers is used for the calculations.
\end{abstract}

Keywords: Monte Carlo; electric vehicles' load; distribution network; renewables; voltage drop

\section{Introduction}

A plethora of studies have been conducted to enhance understanding of electric vehicle connection to the grid [1], and to define expectations for electricity grid performance when distributed generation also plays its own important role [2,3]. These two, relatively new components require wise consideration [4] in order to enhance grids' productivity; this has to be done in a manner which is interconnected [5]. One approach could be electric vehicles that are aggregated to a virtual power plant [6]; in conjunction to renewables generation, the degree to which they are able to increase grid capacity factors is investigated. Moreover, aggregation offers operational benefits to the system operator [7], who is then able to take faster and safer operational decisions. Electric vehicles batteries can present energy storage opportunities for the grid if customer comfort is lightly compromised. Under these conditions, it can provide short-term reserves and offer additional grid flexibility [8].

The increase of available computational power has also transferred to power system applications, thereby advancing the capability of researchers and operators to improve grid performance. This is a one-way path, due to the existing and increasing complexity of the addition of smart devices to the grid. Electric transportation is considered an important factor in this field [9]. Special attention is given to high performance computing (HPC) applications that use probabilistic methods, which are demanding in terms of calculations, but necessary to understand specific phenomena with adequate accuracy. Electric vehicle connections to the grid and the Monte Carlo method is an example [10]. 
However, stochastic methods, and especially Monte Carlo [11], is computationally demanding even for today's standards; hence, specific operational points are developed in this study for a specific network [12].

Electric vehicles charging would also require a grid expansion design factor [13]. Current research on electric vehicles, as far as the distribution network is concerned, gives emphasis to probabilistic methods in order to predict charging patterns, and predict the expected charging behavior. This also affects the connection points for distributed generations, that could be optimally different if connected points are based on probabilistic methods, taking into consideration their intermittency [14]. As far as electric vehicles are concerned, the first step is to characterize their charging demand [15]. In some cases, the system is simulated as a whole, and the operational benefits are optimized based on electric charging owners' behavior [16]. Alternatively, they are connected in a way that relieves distribution system constraints [17]. Active distribution network management could be done by aggregating electric vehicle behavior in a probabilistic manner [12]. Probabilistic studies have also shown good correlation between electric vehicles and renewables [18]. In this research, electric vehicle and load aggregation is performed on the level of a secondary distribution system, at the point of medium voltage (MV)/low voltage (LV) connection, and it is fully controlled.

Having mentioned the above, several studies have researched the emerging phenomenon of electric vehicles. All of them are consumer oriented, giving emphasis to electric vehicles per se, and showing minimal consideration for the electricity grid. On the other hand, the research presented in the current paper is electric grid oriented. It is focused in the procedure of creating optimal electric grid operation points for electric vehicle charging for a real distribution network [19], i.e., as it operates today, based on objective equations, solved with Monte Carlo using high performance computing.

\section{Line under Investigation}

This method is applied to a real representative distribution network [19]. Details of the network are available at the online dataset (http:/ / dx.doi.org/10.7910/DVN/1I6MKU). Source code and data sets are open sourced to all users. This network spans fifty-five kilometers (55 km), a typical size for distribution rural lines, and its total installed load capacity is twelve mega-volt-amperes (12MVA). It is radially organized, and all conductors are optimized based on the expectation that higher currents appear near the feeder at the high to medium voltage substation.

It has forty-five (45) medium-to-low voltage transformers that are used to supply low voltage loads. These are the expected points for the aggregated connection of electric vehicles. The electric vehicles are connected to the secondary distribution network at low voltage levels, i.e., after the medium to low voltage transformers. It is assumed that the maximum load, including electric vehicles', does not exceed the maximum observed active and reactive power of the existing installations. In this manner, the maximum utilization of the network is achieved.

Given that distribution network is limited, solar irradiation does not change significantly across the line. According to this assumption, production from photovoltaic plants could be safely assumed to be similar. Hence, the plants connected to the line under investigation would operate at the same percentage of installed capacity. There are also twenty-four (24) photovoltaic plants with an installed capacity totaling to $6929 \mathrm{MW}$. This network is not connected to any other type of distributed generation. The network in detail is available at [19].

\section{Monte Carlo and Power Flow Methods}

The Monte Carlo method is applied to numerically stochastic processes, and is used to simulate probabilistic physical phenomena. For this research, it is assumed that each of the forty-five (45) loading nodes of the line under consideration in this analysis can independently receive an active load Sn of up to 1 pu of its capacity:

$$
0 \leq S_{1} \ldots S_{45} \leq 1 \mathrm{pu}
$$


To achieve this input, a one-dimension table with 45 rows is defined that is assigned continuous random variables with values from 0 to 1 . According to the following definition, a continuous random variable $x$ has the properties of the function:

$$
\int_{-\infty}^{\infty} f(x) d x=F(\infty)=1
$$

In other words, probability is uniform across all applicable values. However, in the reality of modern computing, absolute random numbers cannot be produced. Pseudorandom numbers are used, with satisfactory results. Pseudorandom number also demonstrate additional research related benefits. The same procedure has always been used to produce them, i.e., they are always the same for a given application. Therefore, results are replicable, hence better benchmarked and controlled.

AC power flow is the typical procedure for solving problems of power systems steady state analysis, which is also applied to this research [20]; it is a widely used and well-known procedure [21]. A typical element has the following admittance $Y i j$ :

$$
Y_{i j}=\left|Y_{i j}\right|<\Theta_{i j}=\left|Y_{i j}\right| \cos \Theta_{i j}+j\left|Y_{i j}\right| \sin \Theta_{i j}=G_{i j}+j B_{i j}
$$

and the voltage of a given bus is given as:

$$
V_{i}=\left|V_{i}\right|<\delta_{i}=\left|V_{i}\right| \cos \delta_{i}+j\left|V_{i}\right| \sin \delta_{i}
$$

the current of this bus is given from:

$$
I_{i}=Y_{i 1} V_{1}+Y_{i 2} V_{2}+\ldots+Y_{i n} V_{n}=\sum_{n=1}^{N} Y_{i n} V_{n}
$$

and its active and reactive power:

$$
P_{i}+j Q_{j}=V_{i}^{*} \sum_{n=1}^{N} Y_{i n} V_{n}
$$

which makes:

$$
\begin{gathered}
P_{i}+j Q_{j}=\sum_{n=1}^{N}\left|Y_{i n} V_{n} V_{i}\right|<\left(\Theta_{i n}+\delta_{n}-\delta_{i}\right) \\
P_{i}=\sum_{n=1}^{N}\left|Y_{i n} V_{n} V_{i}\right| \cos \left(\Theta_{i n}+\delta_{n}-\delta_{i}\right) \\
Q_{i}=\sum_{n=1}^{N}\left|Y_{i n} V_{n} V_{i}\right| \sin \left(\Theta_{i n}+\delta_{n}-\delta_{i}\right)
\end{gathered}
$$

at every time the scheduled power needs to be similar to the calculated:

$$
\begin{gathered}
\Delta P_{i}=P_{i, \text { sch }}-P_{i, \text { calc }}=\left(P_{g i}-P_{d i}\right)-P_{i, \text { calc }} \\
\Delta Q_{i}=Q_{i, \text { sch }}-Q_{i, \text { calc }}=\left(Q_{g i}-Q_{d i}\right)-Q_{i, \text { calc }}
\end{gathered}
$$

and consequently

$$
\begin{gathered}
g_{i^{\prime}}=P_{i}-P_{i, s c h}=P_{i}-\left(P_{g i}-P_{d i}\right)=0 \\
g_{i^{\prime \prime}}=Q_{i}-Q_{i, s c h}=Q_{i}-\left(Q_{g i}-Q_{d i}\right)=0
\end{gathered}
$$

the total active power loss is calculated by subtracting from the total generation the total load

$$
P_{L}=\sum_{i=1}^{N} P_{i}=\sum_{i=1}^{N} P_{g i}-\sum_{i=1}^{N} P_{d i}
$$


and similarly, for the reactive power

$$
\sum_{i=1}^{N} Q_{i}=\sum_{i=1}^{N} Q_{g i}-\sum_{i=1}^{N} Q_{d i}
$$

Newton-Raphson is the numerical method used to solve the above-mentioned equations. A short description is provided below. If two equations are considered:

$$
\begin{aligned}
& g_{1}\left(x_{1}, x_{2}, u\right)=h_{1}\left(x_{1}, x_{2}, u\right)-b_{1}=0 \\
& g_{2}\left(x_{1}, x_{2}, u\right)=h_{2}\left(x_{1}, x_{2}, u\right)-b_{2}=0
\end{aligned}
$$

then, the solutions $x_{1}^{*}$ and $x_{2}^{*}$ can be yielded from:

$$
\begin{aligned}
& g_{1}\left(x_{1}^{*}, x_{2}^{*}, u\right)=g_{1}\left(x_{1}^{(0)}+\Delta x_{1}^{(0)}, x_{2}^{(0)}+\Delta x_{2}^{(0)}, u\right)=0 \\
& g_{2}\left(x_{1}^{*}, x_{2}^{*}, u\right)=g_{2}\left(x_{1}^{(0)}+\Delta x_{1}^{(0)}, x_{2}^{(0)}+\Delta x_{2}^{(0)}, u\right)=0
\end{aligned}
$$

And then expanding in Taylor series:

$$
\begin{aligned}
& g_{1}\left(x_{1}^{*}, x_{2}^{*}, u\right)=g_{1}\left(x_{1}^{(0)}, x_{2}^{(0)}, u\right)+\left.\Delta x_{1}^{(0)} \frac{\partial g_{1}}{\partial x_{1}}\right|^{0}+\left.\Delta x_{2}^{(0)} \frac{\partial g_{1}}{\partial x_{2}}\right|^{0}+\ldots=0 \\
& g_{2}\left(x_{1}^{*}, x_{2}^{*}, u\right)=g_{2}\left(x_{1}^{(0)}, x_{2}^{(0)}, u\right)+\left.\Delta x_{1}^{(0)} \frac{\partial g_{2}}{\partial x_{1}}\right|^{0}+\left.\Delta x_{2}^{(0)} \frac{\partial g_{2}}{\partial x_{2}}\right|^{0}+\ldots=0
\end{aligned}
$$

which can be rewritten as:

$$
\left[\begin{array}{ll}
\frac{\partial g_{1}}{\partial x_{1}} & \frac{\partial g_{1}}{\partial x_{2}} \\
\frac{\partial g_{2}}{\partial x_{1}} & \frac{\partial g_{2}}{\partial x_{2}}
\end{array}\right]^{(0)}\left[\begin{array}{c}
\Delta x_{1}^{(0)} \\
\Delta x_{2}^{(0)}
\end{array}\right]=\left[\begin{array}{l}
0-g_{1}\left(x_{1}^{(0)}, x_{2}^{(0)}, u\right) \\
0-g_{2}\left(x_{1}^{(0)}, x_{2}^{(0)}, u\right)
\end{array}\right]=\left[\begin{array}{c}
b_{1}-h_{1}\left(x_{1}^{(0)}, x_{2}^{(0)}, u\right) \\
b_{2}-h_{2}\left(x_{1}^{(0)}, x_{2}^{(0)}, u\right)
\end{array}\right]
$$

where the Jacobian matrix is:

$$
J^{(0)}=\left[\begin{array}{ll}
\frac{\partial g_{1}}{\partial x_{1}} & \frac{\partial g_{1}}{\partial x_{2}} \\
\frac{\partial g_{2}}{\partial x_{1}} & \frac{\partial g_{2}}{\partial x_{2}}
\end{array}\right]^{(0)}
$$

this gives:

$$
J^{(0)}\left[\begin{array}{l}
\Delta x_{1}^{(0)} \\
\Delta x_{2}^{(0)}
\end{array}\right]=\left[\begin{array}{c}
\Delta g_{1}^{(0)} \\
\Delta g_{2}^{(0)}
\end{array}\right]
$$

and the new estimates are:

$$
\begin{aligned}
& x_{1}^{(1)}=x_{1}^{(0)}+\Delta x_{1}^{(0)} \\
& x_{2}^{(1)}=x_{2}^{(0)}+\Delta x_{2}^{(0)}
\end{aligned}
$$

\section{Simulation Procedure}

The source code for this publication has been simulated on Mathworks Matlab Runtime v92, 2017a [22], compiled on Unix operating system and run on Aris high performance computing [23]. High performance computing was not necessary to achieve the research goals; however, it reduces computational time and provides the capability of scaling up to larger electricity networks. Power load flow analysis is conducted on Matpower 6.0 code [24,25]. The source code and the results are provided in the supplementary materials of this manuscript, available on Harvard Dataverse [26].

Figure 1 presents the flow chart for the proposed calculation method. Initially, the algorithm inputs the distribution network data. This includes information of its load and production nodes connection, 
line characteristics, as well as their impedance and reactance to perform load flow calculations. Then the system creates random loading using the Monte Carlo method [11] for all applicable nodes, and performs load flow analysis.

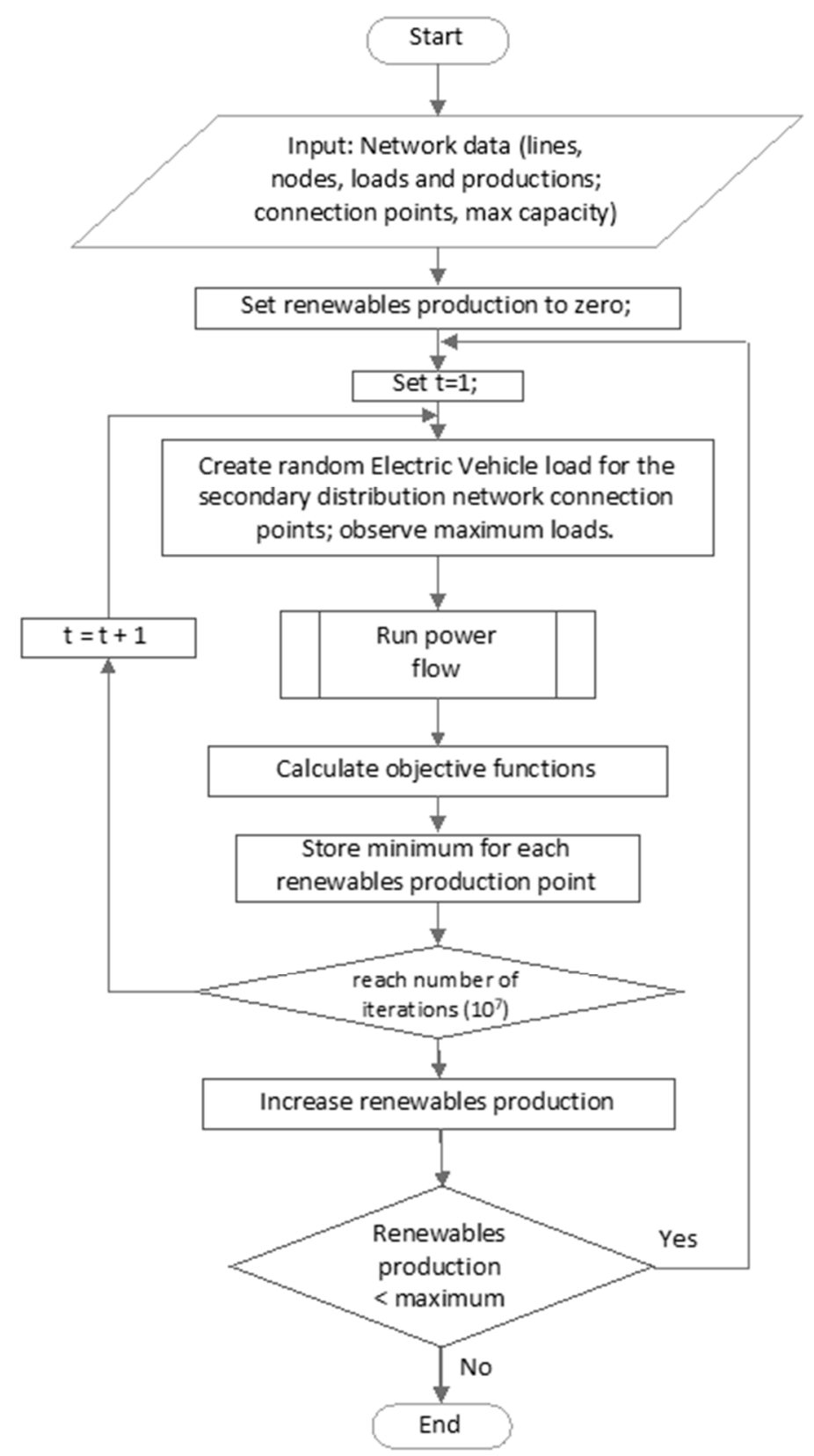

Figure 1. Calculating operational points optimization formula minimums.

The procedure repeats numerous times in order to cover all applicable cases, and the best result is stored for each operational point. An ARIS high performance computer was able to simulate each one of the operational points, using one processor, in $48 \mathrm{~h}$ [23]. Source code total calculation time can be improved if the number of Monte Carlo iterations are reduced, but this could lead to suboptimal results. This source code may also run on personal computers, but usually higher computational times are required.

The default solver uses Newton's method [20]. This type of analysis is adequate to perform load aggregation and production analysis in a steady state. 
The objective functions applied to this research take into consideration the maximum electric vehicle charging load and the minimum voltage across the network, based on the available production from distributed generation. From these two aspects, the charging load is more important to the analysis, and consequently, it receives a higher contribution factor. The final objective is achieved by minimizing the following equations:

$$
\begin{aligned}
& f_{1}(\mathrm{x})=\min _{0 \leq \mathrm{x} \leq 1}\left(0.5 P_{E V}(\mathrm{x})+0.5 V_{\min }(\mathrm{x})\right) \\
& f_{2}(\mathrm{x})=\min _{0 \leq \mathrm{x} \leq 1}\left(0.6 P_{E V}(\mathrm{x})+0.4 V_{\min }(\mathrm{x})\right) \\
& f_{3}(\mathrm{x})=\min _{0 \leq \mathrm{x} \leq 1}\left(0.7 P_{E V}(\mathrm{x})+0.3 V_{\text {min }}(\mathrm{x})\right) \\
& f_{4}(\mathrm{x})=\min _{0 \leq \mathrm{x} \leq 1}\left(0.8 P_{E V}(\mathrm{x})+0.2 V_{\min }(\mathrm{x})\right)
\end{aligned}
$$

where:

Pev stands for the total active load of the line. It is assumed that $\cos \varphi \approx 1$.

$$
P_{e v}=\frac{\sum_{1}^{45} S_{n}}{S_{t o t}}, \text { where } S_{t o t} \text { is the sum of all loads' installed capacity }
$$

and $V_{\min }$ is the minimum voltage observed at any node of the line.

All the above are calculated for increasing production from the connected-to-the-network distributed generation sources, such as photovoltaic plants. Then ten optimal operational points for each optimization equation were created, increasing renewables' production from zero to maximum. These are operational points for a given network based on the availability of energy produced from renewables that meet the above-mentioned objective functions.

\section{Results and Discussion}

Simulation results have shown consistency across all applications to this work's minimization formulas. According to the conducted simulations, there are repeating loading optimization patterns, unique for each line according to the imposed constraints in terms of distributed generation production and minimization requirements.

For $f_{1}(\mathrm{x})$ there are five (5) operational points (Tables 1 and 2) across the installed capacity of the distributed generation connected to the line. For each transformer connection node, the load percentage in conjunction to the maximum observed load has been calculated. It was observed that in some cases, this percentage is very low. This is due to the specific characteristics of the line, and hence, reinforcement is suggested near these transformers.

The exact manner in which this reinforcement shall be done is not currently clear to the researchers. A probabilistic approach can be applied, which can be part of future work. To achieve better performance, it is proposed that messed, instead of radial, topology be applied; however, issues of protection may arise.

For $f_{2}(\mathrm{x})$, there are three (3) operational points (Tables 3 and 4 ) across the installed capacity of the distributed generation connected to the line. To a certain degree, these are similar to the operational points derived from the other equations. This observation further supports the possibility of having line-specific optimal operation points across a wide range of operating conditions. This is to be further investigated. 
Table 1. Optimal loading per node according to $f_{1}(\mathrm{x})$ for increasing production up to $50 \%$ of the distributed generator's installed capacity.

\begin{tabular}{|c|c|c|c|c|c|c|}
\hline \#Transformer & $\begin{array}{c}f_{1}(\mathbf{x}) \text {-without DG } \\
\text { (Percentage of } \\
\text { Maximum Observed } \\
\text { Load) }\end{array}$ & $\begin{array}{c}f_{1}(\mathbf{x})-10 \% \text { DG } \\
\text { (Percentage of } \\
\text { Maximum Observed } \\
\text { Load) }\end{array}$ & $\begin{array}{c}f_{1}(x)-20 \% \text { DG } \\
\text { (Percentage of } \\
\text { Maximum Observed } \\
\text { Load) }\end{array}$ & $\begin{array}{c}f_{1}(\mathbf{x})-30 \% \text { DG } \\
\text { (Percentage of } \\
\text { Maximum Observed } \\
\text { Load) }\end{array}$ & $\begin{array}{c}f_{1}(\mathbf{x})-40 \% \text { DG } \\
\text { (Percentage of } \\
\text { Maximum Observed } \\
\text { Load) }\end{array}$ & $\begin{array}{c}f_{1}(\mathrm{x})-50 \% \text { DG } \\
\text { (Percentage of } \\
\text { Maximum Observed } \\
\text { Load) }\end{array}$ \\
\hline 28 & 0.8382 & 0.0301 & 0.8382 & 0.8177 & 0.3908 & 0.152 \\
\hline 31 & 0.8858 & 0.9023 & 0.8858 & 0.9598 & 0.9425 & 0.885 \\
\hline 39 & 0.9049 & 0.9112 & 0.9049 & 0.8688 & 0.9894 & 0.9194 \\
\hline 45 & 0.9762 & 0.925 & 0.9762 & 0.9796 & 0.9281 & 0.9924 \\
\hline 49 & 0.3472 & 0.9478 & 0.3472 & 0.9235 & 0.9267 & 0.8259 \\
\hline 61 & 0.6871 & 0.9702 & 0.6871 & 0.124 & 0.8382 & 0.478 \\
\hline 74 & 0.8276 & 0.452 & 0.8276 & 0.7596 & 0.1854 & 0.9877 \\
\hline 80 & 0.1646 & 0.6175 & 0.1646 & 0.3251 & 0.2775 & 0.3298 \\
\hline 89 & 0.9733 & 0.8401 & 0.9733 & 0.8711 & 0.844 & 0.8278 \\
\hline 91 & 0.5142 & 0.7913 & 0.5142 & 0.2599 & 0.8462 & 0.8417 \\
\hline 92 & 0.6764 & 0.9131 & 0.6764 & 0.8997 & 0.8684 & 0.9708 \\
\hline 39_18 & 0.9934 & 0.9186 & 0.9934 & 0.8458 & 0.7157 & 0.9316 \\
\hline 39_51 & 0.9075 & 0.9167 & 0.9075 & 0.8253 & 0.9441 & 0.9404 \\
\hline 39_51_3 & 0.8375 & 0.7877 & 0.8375 & 0.3792 & 0.2889 & 0.0673 \\
\hline $39 \_94$ & 0.8407 & 0.5423 & 0.8407 & 0.8235 & 0.9148 & 0.957 \\
\hline $45 \_16 \mathrm{~A}$ & 0.8967 & 0.9474 & 0.8967 & 0.8395 & 0.314 & 0.6439 \\
\hline 45_1A_4 & 0.2702 & 0.6134 & 0.2702 & 0.7997 & 0.8243 & 0.7673 \\
\hline 49_9 & 0.7456 & 0.3974 & 0.7456 & 0.4233 & 0.2874 & 0.9802 \\
\hline 61_10_6 & 0.3127 & 0.9037 & 0.3127 & 0.6078 & 0.9238 & 0.7111 \\
\hline $61 \_28$ & 0.5856 & 0.5735 & 0.5856 & 0.8873 & 0.7583 & 0.1116 \\
\hline 61_28_9 & 0.8431 & 0.887 & 0.8431 & 0.3272 & 0.6855 & 0.9387 \\
\hline 61_32_16 & 0.3256 & 0.8248 & 0.3256 & 0.6824 & 0.5453 & 0.0672 \\
\hline 61_32_162 & 0.4562 & 0.4351 & 0.4562 & 0.5759 & 0.5438 & 0.5693 \\
\hline 61_32_31 & 0.3894 & 0.0361 & 0.3894 & 0.8039 & 0.1964 & 0.6203 \\
\hline 61_32_31_1 & 0.1494 & 0.1645 & 0.1494 & 0.0344 & 0.3047 & 0.533 \\
\hline $61 \_45$ & 0.0755 & 0.6439 & 0.0755 & 0.8028 & 0.9217 & 0.0153 \\
\hline 61_45_12 & 0.8685 & 0.4486 & 0.8685 & 0.9713 & 0.4982 & 0.8222 \\
\hline 61_45_4A_1 & 0.1951 & 0.3019 & 0.1951 & 0.7956 & 0.2272 & 0.879 \\
\hline 61_5 & 0.6946 & 0.9572 & 0.6946 & 0.8117 & 0.619 & 0.9783 \\
\hline 61_6_6A & 0.7501 & 0.1219 & 0.7501 & 0.5161 & 0.2891 & 0.7149 \\
\hline 61_7_2 & 0.7086 & 0.6227 & 0.7086 & 0.5581 & 0.7063 & 0.919 \\
\hline 61_7_2_1A_2 & 0.3583 & 0.8671 & 0.3583 & 0.7513 & 0.4076 & 0.5091 \\
\hline
\end{tabular}


Table 1. Cont.

\begin{tabular}{|c|c|c|c|c|c|c|}
\hline \#Transformer & $\begin{array}{c}f_{1}(\mathbf{x}) \text {-without DG } \\
\text { (Percentage of } \\
\text { Maximum Observed } \\
\text { Load) }\end{array}$ & $\begin{array}{c}f_{1}(\mathrm{x})-10 \% \text { DG } \\
\text { (Percentage of } \\
\text { Maximum Observed } \\
\text { Load) }\end{array}$ & $\begin{array}{c}f_{1}(\mathrm{x})-20 \% \mathrm{DG} \\
\text { (Percentage of } \\
\text { Maximum Observed } \\
\text { Load) }\end{array}$ & $\begin{array}{c}f_{1}(\mathbf{x})-30 \% \text { DG } \\
\text { (Percentage of } \\
\text { Maximum Observed } \\
\text { Load) }\end{array}$ & $\begin{array}{c}f_{1}(\mathrm{x})-40 \% \text { DG } \\
\text { (Percentage of } \\
\text { Maximum Observed } \\
\text { Load) }\end{array}$ & $\begin{array}{c}f_{1}(\mathbf{x})-50 \% \text { DG } \\
\text { (Percentage of } \\
\text { Maximum Observed } \\
\text { Load) }\end{array}$ \\
\hline 61_7_2_2 & 0.2394 & 0.4019 & 0.2394 & 0.6523 & 0.716 & 0.4857 \\
\hline $61 \_7 \_8$ & 0.8612 & 0.9942 & 0.8612 & 0.8626 & 0.8057 & 0.9161 \\
\hline $68 \_3$ & 0.7148 & 0.807 & 0.7148 & 0.6459 & 0.2232 & 0.851 \\
\hline 74_1A_4 & 0.7745 & 0.2404 & 0.7745 & 0.8569 & 0.8363 & 0.7064 \\
\hline 74_1A_4 4 6 & 0.596 & 0.5571 & 0.596 & 0.1776 & 0.5202 & 0.4533 \\
\hline $87 \_3$ & 0.2453 & 0.3263 & 0.2453 & 0.1183 & 0.9159 & 0.8033 \\
\hline 87_3_1 & 0.598 & 0.8487 & 0.598 & 0.12 & 0.8948 & 0.9351 \\
\hline 92_21_4_3 & 0.1793 & 0.6066 & 0.1793 & 0.4977 & 0.1764 & 0.912 \\
\hline 92_21_66 & 0.7871 & 0.5732 & 0.7871 & 0.7446 & 0.7469 & 0.1158 \\
\hline 92_21A & 0.3066 & 0.676 & 0.3066 & 0.8669 & 0.799 & 0.3738 \\
\hline 92_30_1 & 0.201 & 0.5476 & 0.201 & 0.5093 & 0.033 & 0.8454 \\
\hline $92 \_40$ & 0.4673 & 0.3691 & 0.4673 & 0.1872 & 0.2473 & 0.1147 \\
\hline 92_8_1 & 0.9249 & 0.0075 & 0.9249 & 0.247 & 0.4304 & 0.2284 \\
\hline
\end{tabular}

Table 2. Optimal loading per node according to $f_{1}(\mathrm{x})$, for increasing production from to $50 \%$ to full of the distributed generator's installed capacity.

\begin{tabular}{|c|c|c|c|c|c|}
\hline \#Transformer & $\begin{array}{c}f_{1}(\mathbf{x})-60 \% \text { DG, } 70 \% \text { DG, } 80 \% \\
\text { DG, } 90 \% \text { DG and Full DG } \\
\text { Production (Percentage of } \\
\text { Maximum Observed Load) }\end{array}$ & \#Transformer & $\begin{array}{c}f_{1}(\mathrm{x})-60 \% \text { DG, } 70 \% \text { DG, } 80 \% \\
\text { DG, } 90 \% \text { DG and Full DG } \\
\text { Production (Percentage of } \\
\text { Maximum Observed Load) }\end{array}$ & \#Transformer & $\begin{array}{c}f_{1}(\mathrm{x})-60 \% \text { DG, } 70 \% \text { DG, } 80 \% \\
\text { DG, } 90 \% \text { DG and Full DG } \\
\text { Production (Percentage of } \\
\text { Maximum Observed Load) }\end{array}$ \\
\hline 28 & 0.152 & 45_16A & 0.6439 & 61_7_2 & 0.919 \\
\hline 31 & 0.885 & 45_1A_4 & 0.7673 & 61_7_2_1A_2 & 0.5091 \\
\hline 39 & 0.9194 & 49_9 & 0.9802 & 61_7_2_2 & 0.4857 \\
\hline 45 & 0.9924 & 61_10_6 & 0.7111 & $61 \_7 \_8$ & 0.9161 \\
\hline 49 & 0.8259 & 61_28 & 0.1116 & $68 \_3$ & 0.851 \\
\hline 61 & 0.478 & 61_28_9 & 0.9387 & 74_1A_4 & 0.7064 \\
\hline 74 & 0.9877 & 61_32_16 & 0.0672 & 74_1A_4_6 & 0.4533 \\
\hline 80 & 0.3298 & 61_32_162 & 0.5693 & $87 \_3$ & 0.8033 \\
\hline 89 & 0.8278 & 61_32_31 & 0.6203 & 87_3_1 & 0.9351 \\
\hline 91 & 0.8417 & 61_32_31_1 & 0.533 & 92_21_4_3 & 0.912 \\
\hline 92 & 0.9708 & $61+45$ & 0.0153 & $92 \_21 \_66$ & 0.1158 \\
\hline 39 & 0.9316 & 61_45_12 & 0.8222 & $92 \_21 \mathrm{~A}$ & 0.3738 \\
\hline 39_51 & 0.9404 & 61_45_4A_1 & 0.879 & 92_30_1 & 0.8454 \\
\hline 39_51_3 & 0.0673 & $61 \_5$ & 0.9783 & $92 \_40$ & 0.1147 \\
\hline 39 & 0.957 & 61_6_6A & 0.7149 & 92_8_1 & 0.2284 \\
\hline
\end{tabular}


Table 3. Optimal loading per node according to $f_{2}(\mathrm{x})$ for increasing production up to $50 \%$ of the distributed generator's installed capacity.

\begin{tabular}{|c|c|c|c|c|c|}
\hline \#Transformer & $\begin{array}{c}f_{2}(\mathbf{x}) \text {-without DG, } 10 \% \\
\text { DG (Percentage of } \\
\text { Maximum Observed } \\
\text { Load) }\end{array}$ & $\begin{array}{c}f_{2}(\mathrm{x})-20 \% \text { DG, } 30 \% \text { DG } \\
40 \% \text { DG and } 50 \% \text { DG } \\
\text { (Percentage of Maximum } \\
\text { Observed Load) }\end{array}$ & \#Transformer & $\begin{array}{c}f_{2}(\mathbf{x}) \text {-without DG, } 10 \% \\
\text { DG (Percentage of } \\
\text { Maximum Observed } \\
\text { Load) }\end{array}$ & $\begin{array}{c}f_{2}(\mathrm{x})-20 \% \text { DG, } 30 \% \text { DG, } \\
40 \% \text { DG and } 50 \% \text { DG } \\
\text { (Percentage of Maximum } \\
\text { Observed Load) }\end{array}$ \\
\hline 28 & 0.0301 & 0.152 & 61_32_31 & 0.0361 & 0.6203 \\
\hline 31 & 0.9023 & 0.885 & 61_32_31_1 & 0.1645 & 0.533 \\
\hline 39 & 0.9112 & 0.9194 & $61 \_45$ & 0.6439 & 0.0153 \\
\hline 45 & 0.925 & 0.9924 & 61_45_12 & 0.4486 & 0.8222 \\
\hline 49 & 0.9478 & 0.8259 & 61_45_4A_1 & 0.3019 & 0.879 \\
\hline 61 & 0.9702 & 0.478 & 61_5 & 0.9572 & 0.9783 \\
\hline 74 & 0.452 & 0.9877 & 61_6_6A & 0.1219 & 0.7149 \\
\hline 80 & 0.6175 & 0.3298 & 61_7_2 & 0.6227 & 0.919 \\
\hline 89 & 0.8401 & 0.8278 & 61_7_2_1A_2 & 0.8671 & 0.5091 \\
\hline 91 & 0.7913 & 0.8417 & 61_7_2_2 & 0.4019 & 0.4857 \\
\hline 92 & 0.9131 & 0.9708 & 61_7_8 & 0.9942 & 0.9161 \\
\hline 39_18 & 0.9186 & 0.9316 & $6 \overline{8} \_\overline{3}$ & 0.807 & 0.851 \\
\hline $39 \_51$ & 0.9167 & 0.9404 & 74_1A_4 & 0.2404 & 0.7064 \\
\hline 39_51_3 & 0.7877 & 0.0673 & 74_1A_4_6 & 0.5571 & 0.4533 \\
\hline $39 \_94$ & 0.5423 & 0.957 & $87 \_3$ & 0.3263 & 0.8033 \\
\hline $45 \_16 \mathrm{~A}$ & 0.9474 & 0.6439 & 87_3_1 & 0.8487 & 0.9351 \\
\hline 45_1A_4 & 0.6134 & 0.7673 & 92_21_4_3 & 0.6066 & 0.912 \\
\hline 49_9 & 0.3974 & 0.9802 & 92_21_66 & 0.5732 & 0.1158 \\
\hline 61_10_6 & 0.9037 & 0.7111 & $92 \_21 \mathrm{~A}$ & 0.676 & 0.3738 \\
\hline $61 \_28$ & 0.5735 & 0.1116 & 92_30_1 & 0.5476 & 0.8454 \\
\hline 61_28_9 & 0.887 & 0.9387 & $92 \_40$ & 0.3691 & 0.1147 \\
\hline 61_32_16 & 0.8248 & 0.0672 & 92_8_1 & 0.0075 & 0.2284 \\
\hline 61_32_162 & 0.4351 & 0.5693 & & & \\
\hline
\end{tabular}


Table 4. Optimal loading per node according to $f_{2}(\mathrm{x})$ for increasing production from to $50 \%$ to full of the distributed generator's installed capacity.

\begin{tabular}{|c|c|c|c|c|c|}
\hline \#Transformer & $\begin{array}{c}f_{2}(\mathbf{x})-60 \% \text { DG, } 70 \% \text { DG, } \\
80 \% \text { DG and } 90 \% \text { DG } \\
\text { (Percentage of Maximum } \\
\text { Observed Load) }\end{array}$ & $\begin{array}{c}f_{2}(x) \text {-full DG Production } \\
\text { (Percentage of Maximum } \\
\text { Observed Load) }\end{array}$ & \#Transformer & $\begin{array}{c}f_{2}(\mathrm{x})-60 \% \text { DG, } 70 \% \text { DG, } \\
80 \% \text { DG and } 90 \% \text { DG } \\
\text { (Percentage of Maximum } \\
\text { Observed Load) }\end{array}$ & $\begin{array}{c}f_{2}(\mathbf{x}) \text {-Full DG Production } \\
\text { (Percentage of Maximum } \\
\text { Observed Load) }\end{array}$ \\
\hline 28 & 0.152 & 0.5311 & 61_32_31 & 0.6203 & 0.3476 \\
\hline 31 & 0.885 & 0.9051 & 61_32_31_1 & 0.533 & 0.8526 \\
\hline 39 & 0.9194 & 0.968 & 61_45 & 0.0153 & 0.881 \\
\hline 45 & 0.9924 & 0.945 & 61_45_12 & 0.8222 & 0.5916 \\
\hline 49 & 0.8259 & 0.6184 & 61_45_4A_1 & 0.879 & 0.7937 \\
\hline 61 & 0.478 & 0.6175 & $61 \_5$ & 0.9783 & 0.5695 \\
\hline 74 & 0.9877 & 0.0504 & 61_6_6A & 0.7149 & 0.5387 \\
\hline 80 & 0.3298 & 0.9166 & 61_7_2 & 0.919 & 0.6669 \\
\hline 89 & 0.8278 & 0.8561 & 61_7_2_1A_2 & 0.5091 & 0.765 \\
\hline 91 & 0.8417 & 0.9359 & 61_7_2_2 & 0.4857 & 0.8833 \\
\hline 92 & 0.9708 & 0.8999 & 61_7_8 & 0.9161 & 0.9672 \\
\hline 39_18 & 0.9316 & 0.915 & $68 \_3$ & 0.851 & 0.537 \\
\hline 39_51 & 0.9404 & 0.9189 & 74_1A_4 & 0.7064 & 0.3431 \\
\hline 39_51_3 & 0.0673 & 0.223 & 74_1A_4_6 & 0.4533 & 0.0839 \\
\hline $39 \_94$ & 0.957 & 0.7597 & 87_3 & 0.8033 & 0.4673 \\
\hline 45_16A & 0.6439 & 0.9474 & 87_3_1 & 0.9351 & 0.65 \\
\hline 45_1A_4 & 0.7673 & 0.9928 & 92_21_4_3 & 0.912 & 0.4871 \\
\hline 49_9 & 0.9802 & 0.9317 & $92 \_21 \_66$ & 0.1158 & 0.8276 \\
\hline 61_10_6 & 0.7111 & 0.5847 & $92 \_21 \mathrm{~A}$ & 0.3738 & 0.9753 \\
\hline 61_28 & 0.1116 & 0.0368 & 92_30_1 & 0.8454 & 0.4514 \\
\hline 61_28_9 & 0.9387 & 0.3133 & $92 \_40$ & 0.1147 & 0.969 \\
\hline 61_32_16 & 0.0672 & 0.1616 & 92_8_1 & 0.2284 & 0.7028 \\
\hline 61_32_162 & 0.5693 & 0.7703 & & & \\
\hline
\end{tabular}


For $f_{3}(\mathrm{x})$, there are three (3) operational points (Table 5) across the installed capacity of the distributed generation connected to the line.

Table 5. Optimal loading per node according to $f_{3}(\mathrm{x})$ for increasing production up to the distributed generator's installed capacity.

\begin{tabular}{|c|c|c|c|}
\hline \#Transformer & $\begin{array}{c}f_{3}(\mathbf{x}) \text {-without DG } \\
\text { (Percentage of Maximum } \\
\text { Observed Load) }\end{array}$ & $\begin{array}{c}f_{3}(\mathbf{x})-10 \% \text { DG, } 20 \% \text { DG, } \\
30 \% \text { DG and } 40 \% \text { DG } \\
\text { (Percentage of Maximum } \\
\text { Observed Load) }\end{array}$ & $\begin{array}{c}f_{3}(x)-50 \% \text { DG }, 60 \% \text { DG, } 70 \% \\
\text { DG, } 80 \% \text { DG, } 90 \% \text { DG and Full } \\
\text { DG Production (Percentage of } \\
\text { Maximum Observed Load) }\end{array}$ \\
\hline 28 & 0.0301 & 0.152 & 0.5311 \\
\hline 31 & 0.9023 & 0.885 & 0.9051 \\
\hline 39 & 0.9112 & 0.9194 & 0.968 \\
\hline 45 & 0.925 & 0.9924 & 0.945 \\
\hline 49 & 0.9478 & 0.8259 & 0.6184 \\
\hline 61 & 0.9702 & 0.478 & 0.6175 \\
\hline 74 & 0.452 & 0.9877 & 0.0504 \\
\hline 80 & 0.6175 & 0.3298 & 0.9166 \\
\hline 89 & 0.8401 & 0.8278 & 0.8561 \\
\hline 91 & 0.7913 & 0.8417 & 0.9359 \\
\hline 92 & 0.9131 & 0.9708 & 0.8999 \\
\hline 39_18 & 0.9186 & 0.9316 & 0.915 \\
\hline 39_51 & 0.9167 & 0.9404 & 0.9189 \\
\hline 39_51_3 & 0.7877 & 0.0673 & 0.223 \\
\hline 39_94 & 0.5423 & 0.957 & 0.7597 \\
\hline 45_16A & 0.9474 & 0.6439 & 0.9474 \\
\hline 45_1A_4 & 0.6134 & 0.7673 & 0.9928 \\
\hline 49_9 & 0.3974 & 0.9802 & 0.9317 \\
\hline 61_10_6 & 0.9037 & 0.7111 & 0.5847 \\
\hline $6 \overline{1} \_28$ & 0.5735 & 0.1116 & 0.0368 \\
\hline 61_28_9 & 0.887 & 0.9387 & 0.3133 \\
\hline 61_32_16 & 0.8248 & 0.0672 & 0.1616 \\
\hline 61_32_162 & 0.4351 & 0.5693 & 0.7703 \\
\hline 61_32_31 & 0.0361 & 0.6203 & 0.3476 \\
\hline 61_32_31_1 & 0.1645 & 0.533 & 0.8526 \\
\hline 61_45 & 0.6439 & 0.0153 & 0.881 \\
\hline 61_45_12 & 0.4486 & 0.8222 & 0.5916 \\
\hline 61_45_4A_1 & 0.3019 & 0.879 & 0.7937 \\
\hline 61_5 & 0.9572 & 0.9783 & 0.5695 \\
\hline 61_6_6A & 0.1219 & 0.7149 & 0.5387 \\
\hline 61_-7_2 & 0.6227 & 0.919 & 0.6669 \\
\hline 61_7_2_1A_2 & 0.8671 & 0.5091 & 0.765 \\
\hline 61_7_2_2 & 0.4019 & 0.4857 & 0.8833 \\
\hline $61 \_7 \_8$ & 0.9942 & 0.9161 & 0.9672 \\
\hline $68 \_3$ & 0.807 & 0.851 & 0.537 \\
\hline 74_1A_4 & 0.2404 & 0.7064 & 0.3431 \\
\hline 74_1A_4__6 & 0.5571 & 0.4533 & 0.0839 \\
\hline 87_3 & 0.3263 & 0.8033 & 0.4673 \\
\hline 87_3_1 & 0.8487 & 0.9351 & 0.65 \\
\hline 92_21_4_3 & 0.6066 & 0.912 & 0.4871 \\
\hline $92 \_21 \_66$ & 0.5732 & 0.1158 & 0.8276 \\
\hline $9 \overline{2} \_21 \mathrm{~A}$ & 0.676 & 0.3738 & 0.9753 \\
\hline 92_30_1 & 0.5476 & 0.8454 & 0.4514 \\
\hline $92 \_40$ & 0.3691 & 0.1147 & 0.969 \\
\hline 92_8_1 & 0.0075 & 0.2284 & 0.7028 \\
\hline
\end{tabular}

For $f_{4}(\mathrm{x})$ there are two (2) operational points (Table 6) across the installed capacity of the distributed generation connected to the line. 
Table 6. Optimal loading per node according to $f_{4}(\mathrm{x})$ for increasing production up to the distributed generator's installed capacity.

\begin{tabular}{|c|c|c|c|}
\hline \#Transfomer & $\begin{array}{c}f_{4}(x) \text {-without DG, } 10 \% \text { DG, } 20 \% \\
\text { DG, } 30 \% \text { DG, } 40 \% \text { DG, } 50 \% \text { DG } \\
\text { and } 60 \% \text { DG (Percentage of } \\
\text { Maximum Observed Load) }\end{array}$ & $\begin{array}{c}f_{4}(\mathbf{x})-70 \% \text { DG, } 80 \% \text { DG and } \\
90 \% \text { DG (Percentage of } \\
\text { Maximum Observed Load) }\end{array}$ & $\begin{array}{l}f_{4}(x) \text {-Full DG Production } \\
\text { (Percentage of Maximum } \\
\text { Observed Load) }\end{array}$ \\
\hline 28 & 0.5311 & 0.152 & 0.5311 \\
\hline 31 & 0.9051 & 0.885 & 0.9051 \\
\hline 39 & 0.968 & 0.9194 & 0.968 \\
\hline 45 & 0.945 & 0.9924 & 0.945 \\
\hline 49 & 0.6184 & 0.8259 & 0.6184 \\
\hline 61 & 0.6175 & 0.478 & 0.6175 \\
\hline 74 & 0.0504 & 0.9877 & 0.0504 \\
\hline 80 & 0.9166 & 0.3298 & 0.9166 \\
\hline 89 & 0.8561 & 0.8278 & 0.8561 \\
\hline 91 & 0.9359 & 0.8417 & 0.9359 \\
\hline 92 & 0.8999 & 0.9708 & 0.8999 \\
\hline 39_18 & 0.915 & 0.9316 & 0.915 \\
\hline 39_51 & 0.9189 & 0.9404 & 0.9189 \\
\hline 39_51_3 & 0.223 & 0.0673 & 0.223 \\
\hline 39_94 & 0.7597 & 0.957 & 0.7597 \\
\hline 45_16A & 0.9474 & 0.6439 & 0.9474 \\
\hline 45_1A_4 & 0.9928 & 0.7673 & 0.9928 \\
\hline 49_9 & 0.9317 & 0.9802 & 0.9317 \\
\hline 61_10_6 & 0.5847 & 0.7111 & 0.5847 \\
\hline $6 \overline{1} \_28$ & 0.0368 & 0.1116 & 0.0368 \\
\hline 61_28_9 & 0.3133 & 0.9387 & 0.3133 \\
\hline 61_32_16 & 0.1616 & 0.0672 & 0.1616 \\
\hline 61_32_162 & 0.7703 & 0.5693 & 0.7703 \\
\hline 61_32_31 & 0.3476 & 0.6203 & 0.3476 \\
\hline 61_32_31_1 & 0.8526 & 0.533 & 0.8526 \\
\hline 61_45 & 0.881 & 0.0153 & 0.881 \\
\hline 61_45_12 & 0.5916 & 0.8222 & 0.5916 \\
\hline 61_45_4A_1 & 0.7937 & 0.879 & 0.7937 \\
\hline 61_5 & 0.5695 & 0.9783 & 0.5695 \\
\hline 61_6_6A & 0.5387 & 0.7149 & 0.5387 \\
\hline 61_-7_2 & 0.6669 & 0.919 & 0.6669 \\
\hline 61_7_2_1A_2 & 0.765 & 0.5091 & 0.765 \\
\hline 61_7_2_2 & 0.8833 & 0.4857 & 0.8833 \\
\hline 61_7_8 & 0.9672 & 0.9161 & 0.9672 \\
\hline $68 \_\overline{3}$ & 0.537 & 0.851 & 0.537 \\
\hline 74_1A_4 & 0.3431 & 0.7064 & 0.3431 \\
\hline 74_1A_4_6 & 0.0839 & 0.4533 & 0.0839 \\
\hline 87_3 & 0.4673 & 0.8033 & 0.4673 \\
\hline 87_3_1 & 0.65 & 0.9351 & 0.65 \\
\hline 92_21_4_3 & 0.4871 & 0.912 & 0.4871 \\
\hline 92_21_66 & 0.8276 & 0.1158 & 0.8276 \\
\hline $92-21 \mathrm{~A}$ & 0.9753 & 0.3738 & 0.9753 \\
\hline 92_30_1 & 0.4514 & 0.8454 & 0.4514 \\
\hline $92 \_40$ & 0.969 & 0.1147 & 0.969 \\
\hline $92 \_8 \_1$ & 0.7028 & 0.2284 & 0.7028 \\
\hline
\end{tabular}

It must be noted that, even if the equation results show an increasing value, the optimal line operation points remain, to a certain degree, of the same value. It appears that these are unique for each line, and can be pre-calculated. System operators, being able to affect electric vehicles' load, can adjust grid's operation near to these points. Grid reinforcements can be constructed on the objective of optimizing the optimization points, thereby achieving even better line performance when electric vehicles are to be connected. Moreover, in an effort to validate the obtained results, a genetic algorithm is applied in order to minimize the objective functions (25)-(28). Genetic algorithms are widely applied in science and engineering for solving practical search and optimization problems. The same algorithm gives excellent results in several other optimization problems [27-29]. The obtained results of the two 
applied methodologies (Tables 7 and 8) present adequate convergence, confirming the appropriateness of the proposed methodology.

Table 7. Minimization results for $f_{1}(\mathrm{x})$ and $f_{2}$.

\begin{tabular}{lcclcc}
\hline & Monte Carlo & GA & & Monte Carlo & GA \\
\hline$f_{1}(\mathrm{x})$-without DG & 0.7683 & 0.7944 & $f_{2}(\mathrm{x})$-without DG & 0.7598 & 0.7865 \\
$f_{1}(\mathrm{x})-10 \%$ DG & 0.7872 & 0.7942 & $f_{2}(\mathrm{x})-10 \%$ DG & 0.7756 & 0.7857 \\
$f_{1}(\mathrm{x})-20 \%$ DG & 0.7997 & 0.7958 & $f_{2}(\mathrm{x})-20 \%$ DG & 0.7873 & 0.7926 \\
$f_{1}(\mathrm{x})-30 \%$ DG & 0.8097 & 0.7997 & $f_{2}(\mathrm{x})-30 \%$ DG & 0.7981 & 0.7918 \\
$f_{1}(\mathrm{x})-40 \%$ DG & 0.8175 & 0.8265 & $f_{2}(\mathrm{x})-40 \%$ DG & 0.8073 & 0.8117 \\
$f_{1}(\mathrm{x})-50 \%$ DG & 0.8261 & 0.8346 & $f_{2}(\mathrm{x})-50 \%$ DG & 0.8155 & 0.8316 \\
$f_{1}(\mathrm{x})-60 \%$ DG & 0.8351 & 0.8487 & $f_{2}(\mathrm{x})-60 \%$ DG & 0.8226 & 0.8500 \\
$f_{1}(\mathrm{x})-70 \%$ DG & 0.843 & 0.8346 & $f_{2}(\mathrm{x})-70 \%$ DG & 0.829 & 0.8328 \\
$f_{1}(\mathrm{x})-80 \%$ DG & 0.8499 & 0.8425 & $f_{2}(\mathrm{x})-80 \%$ DG & 0.8345 & 0.8329 \\
$f_{1}(\mathrm{x})-90 \%$ DG & 0.8521 & 0.8523 & $f_{2}(\mathrm{x})-90 \%$ DG & 0.8362 & 0.8439 \\
$f_{1}(\mathrm{x})-$ full DG production & 0.8537 & 0.8748 & $f_{2}(\mathrm{x})$-full DG production & 0.8398 & 0.8728 \\
\hline
\end{tabular}

Table 8. Minimization results for $f_{3}(\mathrm{x})$ and $f_{4}(\mathrm{x})$.

\begin{tabular}{lcclcc}
\hline & Monte Carlo & GA & & Monte Carlo & GA \\
\hline$f_{3}(\mathrm{x})$-without DG & 0.7598 & 0.7821 & $f_{4}(\mathrm{x})$-without DG & 0.7694 & 0.7694 \\
$f_{3}(\mathrm{x})-10 \%$ DG & 0.7741 & 0.7787 & $f_{4}(\mathrm{x})-10 \%$ DG & 0.7786 & 0.7786 \\
$f_{3}(\mathrm{x})-20 \%$ DG & 0.7837 & 0.7805 & $f_{4}(\mathrm{x})-20 \%$ DG & 0.7858 & 0.7858 \\
$f_{3}(\mathrm{x})-30 \%$ DG & 0.7918 & 0.7799 & $f_{4}(\mathrm{x})-30 \%$ DG & 0.7918 & 0.7918 \\
$f_{3}(\mathrm{x})-40 \%$ DG & 0.7987 & 0.8012 & $f_{4}(\mathrm{x})-40 \%$ DG & 0.7969 & 0.7969 \\
$f_{3}(\mathrm{x})-50 \%$ DG & 0.8053 & 0.8133 & $f_{4}(\mathrm{x})-50 \%$ DG & 0.8014 & 0.8014 \\
$f_{3}(\mathrm{x})-60 \%$ DG & 0.8111 & 0.8271 & $f_{4}(\mathrm{x})-60 \%$ DG & 0.8052 & 0.8052 \\
$f_{3}(\mathrm{x})-70 \%$ DG & 0.8162 & 0.8099 & $f_{4}(\mathrm{x})-70 \%$ DG & 0.829 & 0.829 \\
$f_{3}(\mathrm{x})-80 \%$ DG & 0.8207 & 0.8123 & $f_{4}(\mathrm{x})-80 \%$ DG & 0.8345 & 0.8345 \\
$f_{3}(\mathrm{x})-90 \%$ DG & 0.8247 & 0.8245 & $f_{4}(\mathrm{x})-90 \%$ DG & 0.8362 & 0.8362 \\
$f_{3}(\mathrm{x})$-full DG production & 0.8282 & 0.8505 & $f_{4}(\mathrm{x})$-full DG production & 0.8166 & 0.8166 \\
\hline
\end{tabular}

\section{Conclusions}

This analysis provides specific operational points based on the production of the connected distributed generators. Based on these findings, potential charging service providers are able to optimize the charging of electric vehicles connected to the line under investigation. In this way, optimal operations could be achieved. It should be mentioned that it is possible to provide optimization formulas for minimizing line losses or maximizing transferred energy using the provided algorithm.

Loading patterns appear to be consistent across all performed calculations. It is believed that they are connected to the topology of the line, and are to a certain degree unconnected to the load. This is an important observation that requires further investigation.

It is observed that several transformer connection nodes display low percentages of optimal load. To the authors' understanding, these are the areas of the grid that need reinforcement. The reinforcement can be done in a manner for the grid whereby the radial configuration is lost. In this case, new calculations are required.

Simulation results have shown improving performance of the grid for increasing production from distributed generators. This is an expected observation; however, the performed simulations are able to provide quantification.

Future work will include the creation of active protection systems based on pragmatic conditions operational system diagnosis, and further probabilistic analysis for possible reinforcements.

Supplementary Materials: All data and results of this work are available at S. Lazarou, V. Vita, C. Christodoulou and L. Ekonomou, "Replication Data for: Calculating operational patterns for electric vehicle charging on a real distribution network based on renewables' production," Available: http://dx.doi.org/10.7910/DVN/1MWESP and on Researchgate.com.

Author Contributions: All authors contributed equally to the development of this manuscript. 
Funding: The authors acknowledge financial support for the open source publication of this work from the Special Account for Research of ASPETE through the funding program "Strengthening research of ASPETE faculty members" under the project "DECA".

Acknowledgments: This work was supported by computational time granted from the Greek Research \& Technology Network (GRNET) in the National HPC facility -ARIS - under project ID pa171102 [22]. The authors would like to acknowledge researchgate.com and Energies' MDPI reviewers who supported our work providing improvements.

Conflicts of Interest: The authors declare no conflict of interest.

\section{Nomenclature}

\begin{tabular}{|c|c|}
\hline $\mathrm{Lh}$ & Branch line length (in km) \\
\hline $\mathrm{R}$ & Branch line Ohmic resistance per $\mathrm{km}$ (in $\Omega / \mathrm{km}$ ) \\
\hline $\mathrm{L}$ & Branch line Reactance per $\mathrm{km}$ (in $\Omega / \mathrm{km}$ ) \\
\hline S1 & First load transformer Complex-Apparent power (in kVA) \\
\hline S2 & Second load transformer Complex-Apparent power (in kVA) \\
\hline S45 & Last load transformer Complex-Apparent power (in kVA) \\
\hline P1, max & First transformer maximum observed load (active power) (in kW) \\
\hline $\mathrm{P} 2$, $\max$ & Second transformer maximum observed load (active power) (in $\mathrm{kW}$ ) \\
\hline $\mathrm{P} 45, \max$ & Last transformer maximum observed load (active power) (in kW) \\
\hline Stot & Total load installed capacity (in kVA) \\
\hline P1 & First photovoltaic plant Active production power (in kW) \\
\hline P2 & Second photovoltaic plant Active production power (in kW) \\
\hline P24 & Last photovoltaic plant Active production power (in $\mathrm{kW}$ ) \\
\hline Pev & Total active load of the line \\
\hline Vmin & Minimum voltage observed at any node \\
\hline $\mathrm{f} \_1(\mathrm{x})$ & First objective function \\
\hline$f \_2(x)$ & Second objective function \\
\hline f_3 (x) & Third objective function \\
\hline $\mathrm{f} \_4(\mathrm{x})$ & Forth objective function \\
\hline
\end{tabular}

\section{References}

1. Morais, H.; Sousa, T.; Vale, Z.; Faria, P. Evaluation of the electric vehicle impact in the power demand curve in a smart grid environment. Energy Convers. Manag. 2014, 82, 268-282. [CrossRef]

2. Fang, X.; Misra, S.; Xue, G.; Yang, D. Smart Grid-The new and improved power grid: A survey. IEEE Commun. Surv. Tutor. 2012, 14, 944-980. [CrossRef]

3. Vita, V.; Alimardan, T.; Ekonomou, L. The impact of distributed generation in the distribution networks' voltage profile and energy losses. In Proceedings of the 9th IEEE European Modelling Symposium on Mathematical Modelling and Computer Simulation, Madrid, Spain, 6-8 October 2015; pp. 260-265.

4. Rahbari, O.; Vafaeipour, M.; Omar, N.; Rosen, M.A.; Hegazy, O.; Timmermans, J.-M.; Heibati, S.; Bossche, P.V.D. An optimal versatile control approach for plug-in electric vehicles to integrate renewable energy sources and smart grids. Energy 2017, 134, 1053-1067. [CrossRef]

5. Farid, A.; Jiang, B.; Muzhikyan, A.; Youcef-Toumi, K. The need for holistic enterprise control assessment methods for the future electricity grid. Renew. Sustain. Energy Rev. 2016, 56, 669-685. [CrossRef]

6. Zakariazadeh, A.; Jadid, S.; Siano, P. Integrated operation of electric vehicles and renewable generation in a smart distribution system. Energy Convers. Manag. 2015, 89, 99-110. [CrossRef]

7. Bhattarai, B.P.; Myers, K.S.; Bak-Jensen, B.; Mendaza, I.D.d.C.; Turk, R.J.; Gentle, J.P. Optimum aggregation of geographically distributed flexible resources in strategic smart-grid/microgrid locations. Electr. Power Energy Syst. 2017, 92, 193-201. [CrossRef]

8. Knezovic, K.; Marinelli, M.; Zecchino, A.; Andersen, P.B.; Traeholt, C. Supporting involvement of electric vehicles in distribution grids: Lowering the barriers for a proactive integration. Energy 2017, 134, 458-468. [CrossRef]

9. Green, R.C.; Wang, L.; Alam, M. Applications and Trends of High Performance Computing for Electric Power Systems: Focusing on Smart Grid. IEEE Trans. Smart Grid 2013, 4, 422-431. [CrossRef] 
10. Procopiou, A.T.; Quirós-Tortós, J.; Ochoa, L.F. HPC-Based Probabilistic Analysis of LV Networks with EVs: Impacts and Control. IEEE Trans. Smart Grid. 2017, 8, 1479-1487. [CrossRef]

11. Kalos, M.H.; Whitlock, P.A. Monte Carlo Methods; WILEY-VCH Verlag: Hoboken, NJ, USA, 2008.

12. Xiang, Y.; Liua, J.; Liu, Y. Optimal active distribution system management considering aggregated plug-in electric vehicles. Electr. Power Syst. Res. 2016, 131, 105-115. [CrossRef]

13. Abdelsamad, S.; Morsi, W.; Sidhu, T. Optimal secondary distribution system design considering plug-inelectric vehicles. Electr. Power Syst. Res. 2016, 130, 266-276. [CrossRef]

14. Sadeghi, M.; Kalantar, M. Multi types DG expansion dynamic planning in distribution system under stochastic conditions using Covariance Matrix Adaptation Evolutionary Strategy and Monte-Carlo simulation. Energy Convers. Manag. 2014, 87, 455-471. [CrossRef]

15. Sun, S.; Yang, Q.; Yan, W. A novel Markov-based temporal-SoC analysis for characterizing PEV charging demand. IEEE Trans. Ind. Inform. 2018, 14, 156-166. [CrossRef]

16. Arias, A.; Granada, M.; Castro, C.A. Optimal probabilistic charging of electric vehicles in distribution systems. IET Electr. Syst. Transp. 2017, 7, 246-251. [CrossRef]

17. Wu, F.; Sioshansi, R. A two-stage stochastic optimization model for scheduling electric vehicle charging loads to relieve distribution-system constraints. Transp. Res. Part B 2017, 102, 55-82. [CrossRef]

18. Wang, L.; Sharkh, S.; Chipperfield, A. Optimal decentralized coordination of electric vehicles and renewable generators in a distribution network using A* search. Electr. Power Energy Syst. 2018, 98, 474-487. [CrossRef]

19. Lazarou, S.; Vita, V.; Ekonomou, L. An open data repository for steady state analysis of a 100-node electricity distribution network with moderate connection of renewable energy sources. Data Brief 2018, 16, 1095-1101. [CrossRef] [PubMed]

20. Zimmerman, R.D.; Murillo-Sanchez, C.E. Matpower 6.0 User's Manual; PSERC: Tempe, AZ, USA, 2016; Available online: http:/ / www.pserc.cornell.edu/matpower/manual.pdf (accessed on 10 September 2018).

21. Grainger, J.J.; Stevenson, W.D. Power System Analysis; McGraw-Hill: New York, NY, USA, 1993.

22. Mathworks. Matlab Runtime. Available online: https://www.mathworks.com/products/compiler/matlabruntime.html (accessed on 10 September 2018).

23. Greek Research and Technology Network (GRNET). Aris High Performance Computing. Available online: https:/ /hpc.grnet.gr/ (accessed on 10 September 2018).

24. Zimmerman, R.D.; Murillo-Sánchez, C.E.; Thomas, R.J. MATPOWER: Steady-State Operations, Planning and Analysis Tools for Power Systems Research and Education. IEEE Trans. Power Syst. 2011, 26, 12-19. [CrossRef]

25. Murillo-Sánchez, C.E.; Zimmerman, R.D.; Anderson, C.L.; Thomas, R.J. Secure Planning and Operations of Systems with Stochastic Sources, Energy Storage and Active Demand. IEEE Trans. Smart Grid 2013, 4, 2220-2229. [CrossRef]

26. Lazarou, S.; Vita, V.; Christodoulou, C.; Ekonomou, L. Replication Data for: Calculating Operational Patterns for Electric Vehicle Charging on a Real Distribution Network Based on Renewables' Production. Available online: http:/ /dx.doi.org/10.7910/DVN/1MWESP (accessed on 10 September 2018).

27. Gonos, I.; Stathopulos, I. Estimation of multi-layer soil parameters using genetic algorithms. IEEE Trans. Power Deliv. 2005, 20, 100-106. [CrossRef]

28. Christodoulou, C.; Gonos, I.; Stathopulos, I. Estimation of the parameters of metal oxide gapless surge arrester equivalent circuit models using genetic algorithm. Electr. Power Syst. Res. 2011, 81, 1881-1886. [CrossRef]

29. Christodoulou, C.A.; Papanikolaou, N.P.; Gonos, I.F. Design of three-phase autonomous PV residential systems with improved power quality. IEEE Trans. Sustain. Energy 2014, 5, 1027-1035. [CrossRef]

(C) 2018 by the authors. Licensee MDPI, Basel, Switzerland. This article is an open access article distributed under the terms and conditions of the Creative Commons Attribution (CC BY) license (http:/ / creativecommons.org/licenses/by/4.0/). 